\title{
Restoring a fractured incisor of a teenager with partial veneer
}

\begin{abstract}
Since trauma is a common incident that adolescents are faced with, the post-traumatic therapeutic solution presents a challenge for the clinicians. This case report demonstrates how a young patient with minimal extended fracture of incisor was rehabilitated with noninvasive adhesive restoration known as partial veneer or chip with Feld spathic porcelain. Those restorations fit into the anatomy and shape of the teeth perfectly and restore only the fractured part which integrates in tissue economy dynamic. Feldspathic porcelain is the material that best reproduces the characteristics of enamel, in terms of surface properties, modulus of elasticity, resistance to fracture and the reproduction of the optical properties of the dental structure, especially at minimal thickness. In this paper we describe the details of the treatment and the final result which was aesthetic and surpass the patient's expectation.
\end{abstract}

Keywords: adolescent, trauma, partial veneer, feldspathic ceramic, stratification, mini invasive restoration
Volume 12 Issue 2 - 202I

\author{
Dalenda Hadyaoui, Yosra Farhat, Imen \\ Kalghoum, Yosra Gasara, Zohra Nouira, \\ Belhassen Harzallah, Mounir Cherif \\ Department of fixed Prosthodontics, Research laboratory of \\ occlusodontics and ceramic prostheses, University of Monastir, \\ Tunisia
}

Correspondence: Dalenda Hadyaoui, Department of fixed Prosthodontics, Research laboratory of occlusodontics and ceramic prostheses, University of Monastir, Monastir, Tunisia, Email dalendahadyaoui@yahoo.fr

Received: February 08, 2021 | Published: March II, 202 I

\section{Introduction}

Nowadays cosmetic needs have a fundamental importance in our society especially among adolescents. As the face is a key feature of physical appearance and the mouth is an important determinant of its attractiveness, having unpleasant teeth will negatively impact the individual's psychology. ${ }^{1-5}$ Therefore Traumatic dental fracture which is common in children and adolescents has been considered a significant problem in youth, for its potential impact on the quality of their life. The psychological and social effect of dental trauma is generally recognized by its consequences affecting social contact, emotional balance and well-being of the child. Treatment options for simple fractures without pulpal and/or periodontal involvement are chosen depending on various factors including the extent of the fracture, the quality and timing of the initial intervention and the presence or absence of dental fragments.

The reattachment of the broken fragment has been widely studied and showed clinically acceptable prognosis in terms of aesthetics and function. However, the broken piece is not always available, in this case direct restoration with composite resin represents a suitable alternative. ${ }^{6}$ This treatment modality requires no or minimal tooth preparation while achieving reliable bond strength values with the use of enamel and dentin adhesive systems. Regarding the aesthetic outcomes, the low durability of this material leads to aesthetic degradation due to color instability. In addition, the degeneration of its organic matrix due to water absorption can be observed, therefore, a regular maintenance and polishing is recommended to prolong its viability. This makes this option to be considered as temporary solution in some cases.

Beside the resin restraints, the immaturity of a teenagers periodentium is not in favor of placing permanent porcelain veneers, owing to the fact that the practitioner should renew the restoration consequently to follow the margin shifting position, which is not practical but when the extent of fracture is far from the margin, the fixed solution is conceivable in the form of partial veneer or chip made by porcelain independently from the gingival collar. As shown in the literature, porcelain greatly mimics the natural structure of dental elements and is a suitable option to avoid the various deficiencies of composite resin. The material has several important characteristics, biological compatibility, sufficient resistance to compression and abrasion, stable reproduction of the optical properties of the dental structure and adherence to the bonding agent and dental substrates. ${ }^{7,8}$

Feldspathic ceramic veneers can be manufacture by different techniques as $\mathrm{CAD} / \mathrm{CAM}$ or layering. In order to obtain veneers the $\mathrm{CAD} / \mathrm{CAM}$ is a useful system that allows the elaboration of monolithic restoration. ${ }^{9}$ This leads to a single ceramic material which usually lacks the most common effect of the incisal third of anterior dentition, so it is recommended to finish monolithic CAD/CAM restorations with layering technique to obtain a tridimensional effect that simplifies the correction of texture and shape ${ }^{2}$ however, Due to their extreme thinness and smallness the use of milling by cad/cam is not the best suited technique for this type of restoration. On the other side layering meets aesthetics expectation by ensuring a flawless integration of the restoration by the use of different shades and the recreation of the edge's translucidities'..$^{7-10}$

This clinical report presents the case of a patient with fractured incisor who was rehabilitated with Feld spathic ceramic chips.

\section{Case report}

A 13-year-old patient presented for a consultation with his parents concerned about the unsightly appearance of his central incisor. The fracture concerned the distal angle of tooth number 11 while he was playing in the schoolyard. It has been restored several times using direct composite materials. The durability of these restorations has never exceeded a few months. Clinical examination reveals an asymptomatic incisor, the vitality test is positive, the percussion and palpation tests are negative. X-ray examination does not reveal any root or endodontic damage. No dyschromia is observed.

At the first visit, intraoral photographs were taken to analyze the cosmetic aspects of the case (Figures 1-4). During the second session, no preparation was necessary, a simple abrasion of the enamel was sufficient to expose the prisms and to remove any residual resin and so to ease bonding. A polyvinyl siloxane impression (ELITE HD+, 
zhermark, France), was made to build the ceramic restoration. In the laboratory, the chip was made by the parallel stratification technique. The pieces were made with a slight over-contour to allow the feldspathic ceramic to be worked without too much risk of fracture. The periphery of the restorations used a translucent ceramic which would take the shade of the underlying tooth (Figure 5). The ceramist uses the die refractory technique (Nori-Vest Zirconia, Kuraray Noritake Dental Inc, Japan).

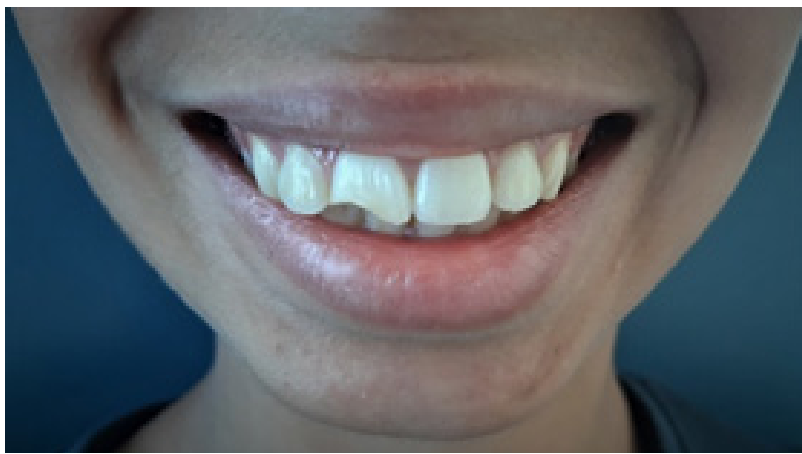

Figure I Patient's smile.

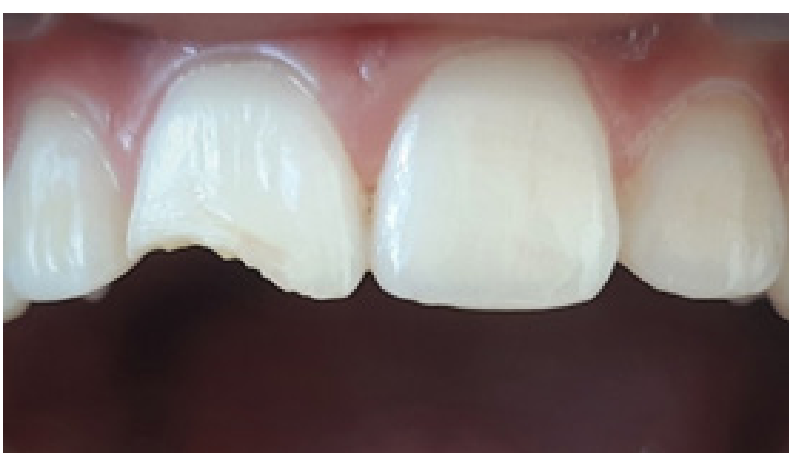

Figure 2 Intraoral front view.

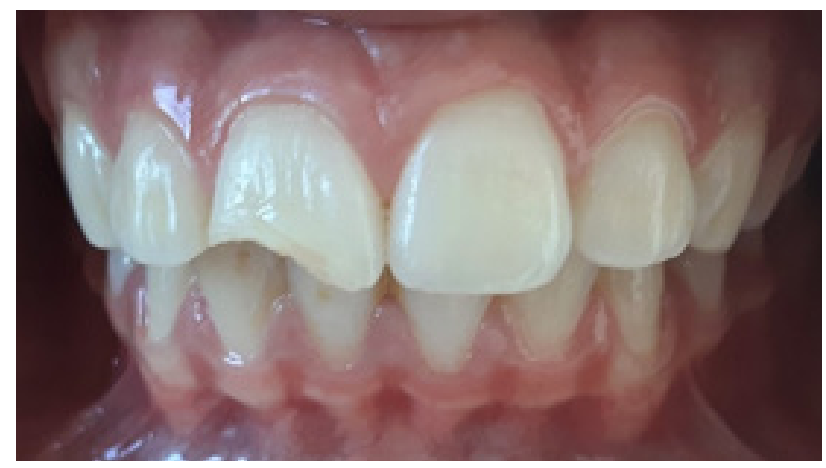

Figure 3 View of the teeth in static occlusion.

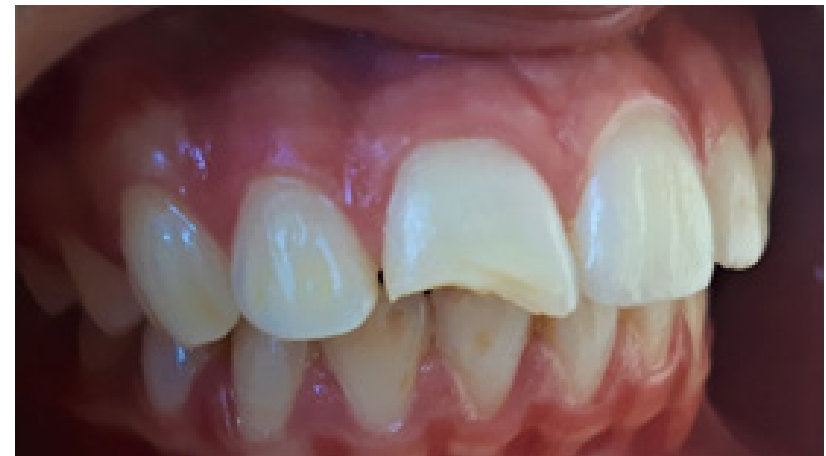

Figure 4 Intraoral right lateral view.

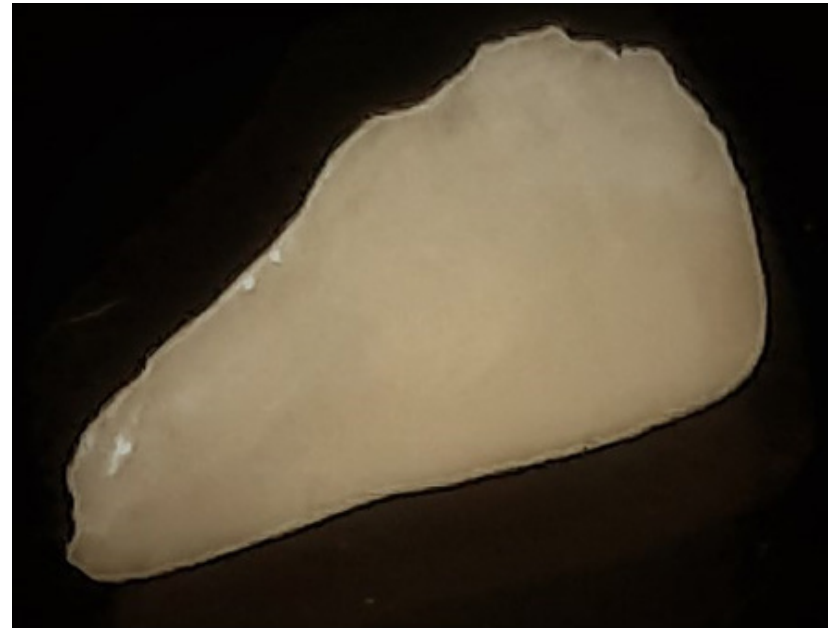

Figure 5 Porcelain partial veneer.

Before bonding, the restorations were evaluated in terms of adaptation (Figure 6). We select the color of the adhesive cement using a try in cement (Variolink II, Ivoclar Vivadent). Once the cement color (color A1) was chosen, pieces were taken for cementation. Firstly, the try-in was removed, washing it under running water, and, subsequently, 10\% hydrofluoric acid was applied (Ceramic Etching Gel, Ivoclar) for 60 seconds. The sample was then washed thoroughly in running water (Figure 7), then silane (Monobond-Ivoclar) was applied for 60 seconds (Figure 8). An operating field was then put in place (Figure 9). The unitary dam is prohibited in order to maintain an overview during bonding. The dental substrate was conditioned with $37 \%$ phosphoric acid (Condac, FGM) for 15 seconds (Figure 10), and was then washed for 45 seconds.

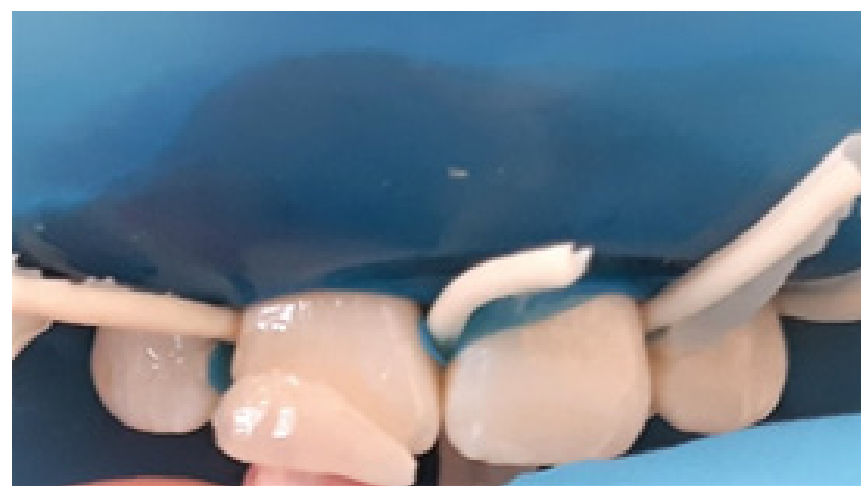

Figure 6 Fitting.

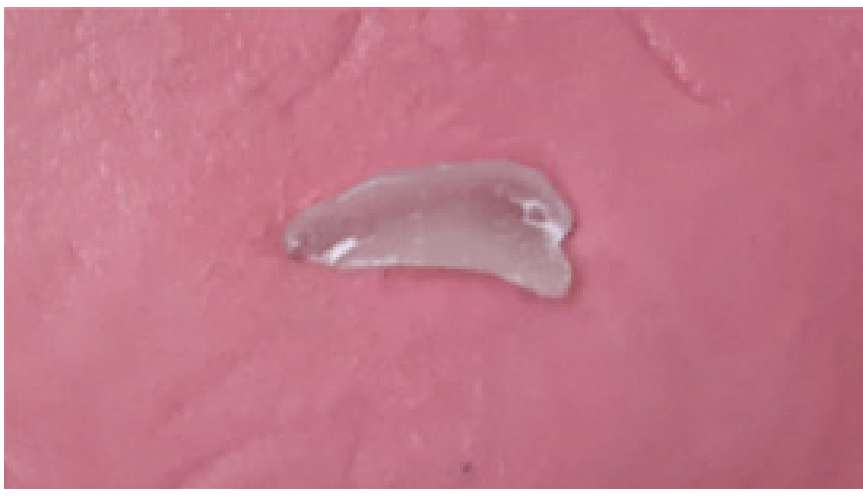

Figure 7 The chip after etching with hydrofluoric acid.

Citation: Hadyaoui D, Farhat Y, Kalghoum I, et al. Restoring a fractured incisor of a teenager with partial veneer.J Dent Health Oral Disord Ther. 2021;I2(2):22-25. DOI: 10.15406/jdhodt.202I.I2.00544 


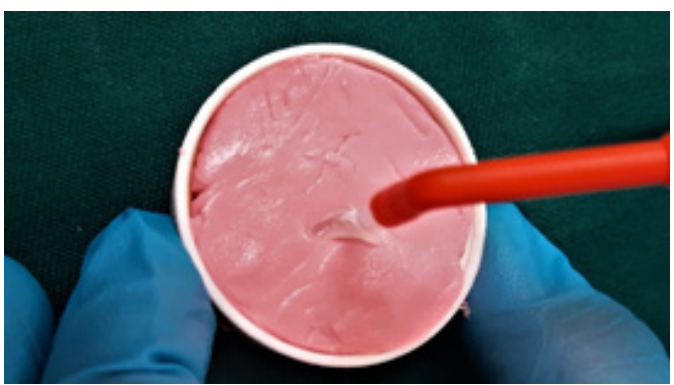

Figure 8 Application if silane.

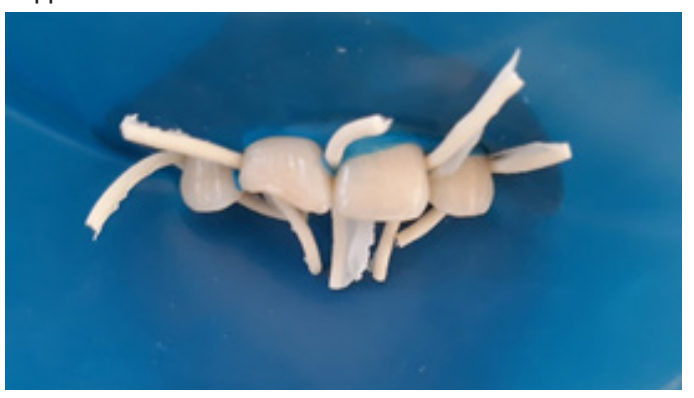

Figure 9 Application of the dam.

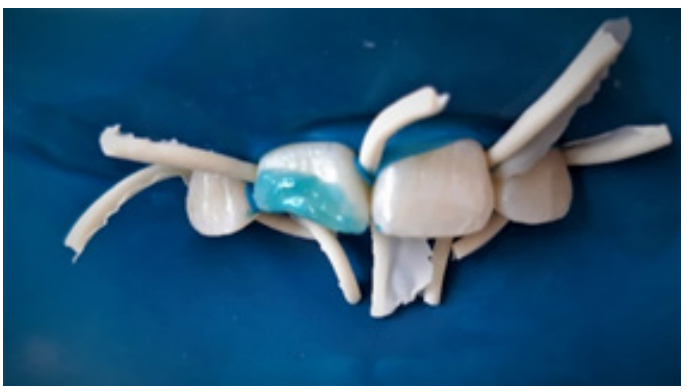

Figure 10 Conditioning of the enamel with $37 \%$.

The adhesive system (Syntac-ivoclar) was applied for 30 seconds, waiting for it to penetrate in the conditioned dentin tubules (Figure 11). A light air jet was applied, and then, the tooth was light cured for 30 seconds. The light-cured resin cement (Variolink II, Ivoclar Vivadent) was activated and placed on the inner side of the pieces that were correctly positioned and photo polymerized for additional two seconds. The excess cement was removed and the curing cycle was terminated (40 seconds on each side). Finally, a glycerin layer (Liquid Strip, Ivoclair Vivadent) was applied between the union piece and tooth, and resin cement was light cured for another 20 seconds, aiming at blocking the oxygen entrance. ${ }^{11,12}$

Final occlusal adjustment was done away from the relative isolation of the operating field (Figure 12). Static occlusion as well as dynamic was controlled (Figure 13).

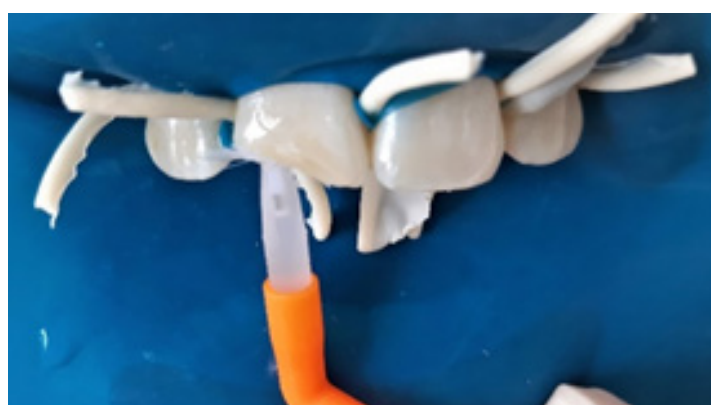

Figure I I Application of adhesive Phosphoric acid for 30 seconds.

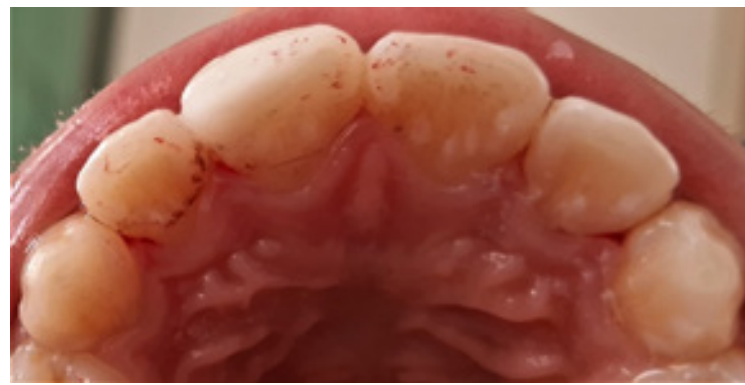

Figure 12 Occlusal adjustment.

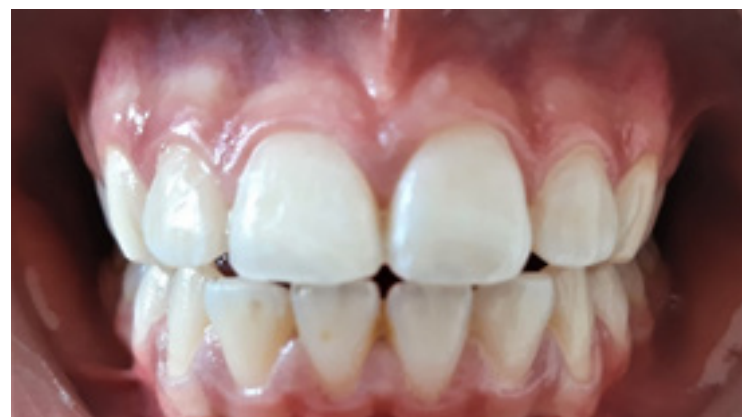

Figure I 3 Propulsion.

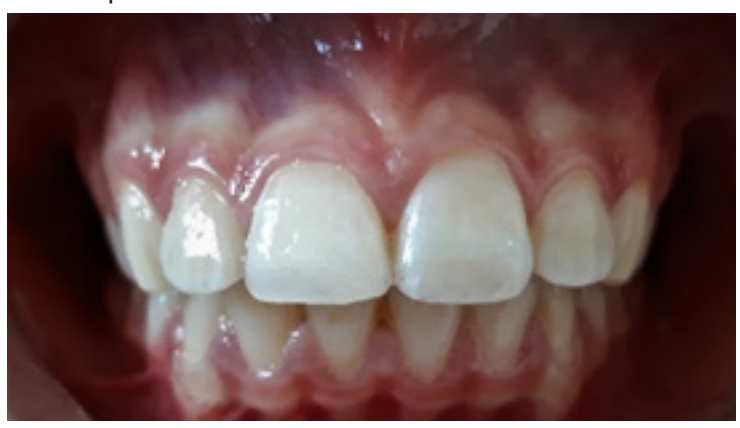

Figure I 4 Final result front view.

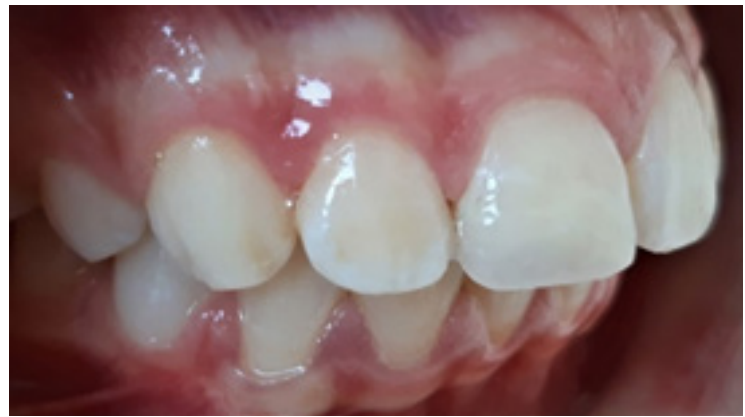

Figure 15 Intra oral lateral view.

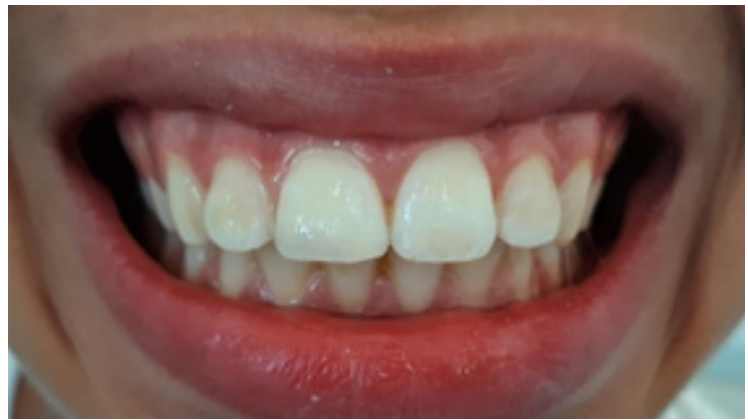

Figure 16 Patient's new smile.

Citation: Hadyaoui D, Farhat Y, Kalghoum I, et al. Restoring a fractured incisor of a teenager with partial veneer.J Dent Health Oral Disord Ther. 202।;I2(2):22-25. DOI: 10.15406/jdhodt.202I.12.00544 


\section{Discussion}

Treating a fractured incisor by a composite resins may initially exceed the patient's assumptions. However, it has a short-term color stability and a low resistance to wear and tear. According to research porcelain veneers, when made in accordance with proper indications and a precise clinical protocol, offer acceptable longevity and appearance. ${ }^{3}$ The no dental preparation allows on the one hand to avoid the realization of temporary veneers and on the other hand to preserve enamel and avoid possible pulp irritation by exposure dentin. Moreover, it is reported that $93 \%$ survival rates for ceramic veneers after 15 years is attributed to the conservation of tooth structure, reliable bonding to enamel, favorable aesthetics, and color stability. Since the high rate of failure in restorations is related to the large exposed dentin, the preparation technique is considered as the most determining factor for the longevity of porcelain laminate veneer. ${ }^{1}$

The choice of Feld spathic ceramic is built on their aesthetics properties and survival rates. The percentages described in the literature are $95.7 \%$ after 5 years and $87 \%$ after 8 years. Katoh et al. ${ }^{13}$ observed that the clinical results of the Feld spathic veneers after 20 years were generally satisfactory. In addition to its undeniable optical characteristics, Feld spathic ceramic offers a wide range of possibilities for layering and partial or total masking of substrate deemed too colored. ${ }^{12}$

The restoration of fractured angle of an incisor requires several layers to restore the opacity of the dentin and translucidity of the insical edge which is not achievable with monolithic restoration by cad/cam system. ${ }^{13,14}$ A study compared the marginal fit of the two techniques by Sorensen et al reported that the vertical marginal discrepancy (for all position combined) for platine foil veneers $(187 \mu \mathrm{m})$ was significantly less than that for veneers made with refractory die technique. ${ }^{4}$

This technique requires having previously used materials with impression of silicone type by addition because their elastic properties which allow them to avoid tears during the numerous casting and demodelings. Once the chip is fabricated, the dentist must handle the partial veneer with great precaution due to their extreme finesse, DEMANGEAT and ARDIC, 2005, recommend the use of stick coated with sticky wax to handle the chip. ${ }^{10}$ Success in the bonding stage depends on the proper preparation, conditioning of the surfaces involved, the ceramic, the dental tissue, and the cementing agent. A partially sectioned rubber dam application is preferred while bonding the veneers. It acts as a physical barrier to oral fluids, moisture, tongue and cheek movements which can reduce the enamel surface energy. ${ }^{15,16}$ The veneer surface should be conditioned with hydrofluoric acid which will not only enhance the micromechanical retentive areas, but also after the surface chemistry to make the silane more effective. Silanization of etched porcelain with a bi-functional coupling agent provides a chemical link between the luting resin composite and porcelain. ${ }^{15}$

\section{Conclusion}

Although there are varieties of therapeutic solutions for the restoration of anterior fractured teeth, the realization of partial veneers or chips makes part of the aesthetic therapeutic solutions which allows to preserve the dental tissues and to guarantie aesthetic integration of the restoration. The procedure was however delicate which makes the collaboration between the dentist and the ceramist essential to achieve success.

\section{References}

1. Alavi AA, Behroozi Z, Eghbal FN. The shear bond strength of porcelain laminate to prepared and unprepared anterior teeth. J Dent Shiraz Univ Med Sci. 2017;18(1):50-55.

2. Durán Ojeda G, Henríquez Gutiérrez I, Guzmán Marusic Á, et al. A stepby-step conservative approach for CAD-CAM laminate veneers. Case Rep Dent. 2017;2017:3801419.

3. Arcelino Farias-Neto, Edna Maria da Cunha Ferreira Gomes, Alfonso Sánchez-Ayala, et al. Esthetic Rehabilitation of the Smile with No-Prep Porcelain Laminates and Partial Veneers. Case Reports in Dentistry. $2015 ; 1-6$

4. Peumans M, Van Meerbeek B, Lambrechts P, et al. Porcelain veneers: a review of the literature. J Dent. 2000;28(3):163-177.

5. Xiao Deng, Yun-ji Wang, Feng Deng, et al. Psychological well-being, dental esthetics, and psychosocial impacts in adolescent orthodontic patients: A prospective longitudinal study. Am J Orthod Dentofacial Orthop. 153(1):87-96.e2.

6. Guido Batalocco, Heeje Lee, Carlo Ercoli, et al. Fracture resistance of composite resin restorations and porcelain veneers in relation to residual tooth structure in fractured incisors. Dent Traumatol. 2012;28(1):75-80.

7. Feimin Zhang, Guido Heydecke, Michael E Razzoog. Double-layer porcelain veneers: Effect of layering on resulting veneer color. The Journal of Prosthetic Dentistry. 84(4):425-431.

8. Tassiana Cançado Melo Sá, Monize Ferreira Figueiredo de Carvalho, Julio Celso M. de Sá, et al. Esthetic rehabilitation of anterior teeth with different thicknesses of porcelain laminate veneers: An 8 year follow up clinical evaluation. J Dent. 12(4):590.

9. Haralur SB. Microleakage of porcelain laminate veneers cemented with different bonding techniques. J Clin Exp Dent. 2018; 10(2):166-171.

10. Romain Demangeat AA. Facettes partielles ultra pelliculaires en céramique. Paris: Quintessence International, 2005.

11. Oztürk E, Bolay S. Survival of porcelain Laminate Veneers with different degrees of dentin exposure: 2-year clinical results. J Adhes Dent. 2014;16(5):481-489.

12. Cente Faus-Matoses, Ignacio Faus-Matoses, Esther Ruiz-Bell, et al. Severe tetracycline dental discoloration: Restoration with conventional feldspathic ceramic veneers. J Clin Exp Dent. 2017;9(11):e1379-1382.

13. Katoh Y, Taira Y, Kato C, et al. A case report of a 20-year clinical follow-up of porcelain laminate veneer restorations. Oper Dent. 2009;34(5):626-630.

14. Gary M Radz. Minimum Thickness Anterior Porcelain Restorations. Dent Clin North Am. 2011;55(2):353-370.

15. Peumans M, Van Meerbeek B, Yoshida Y, et al. Porcelain veneers bonded to tooth structure: an ultra-morphological FESEM examination of the adhesive interface. Dent Mater. 1999;15:105-119.

16. Edelhoff D, Prandtner O, Saeidi Pour R, et al. Anterior restorations: the performance of ceramic veneers. Quintescence Int. 2018;49(2):89-101. 